\title{
Real-Life Effectiveness of Vismodegib in Patients with Metastatic and Advanced Basal Cell Carcinoma: Characterization of Adverse Events and Assessment of Health-Related Quality of Life using the Dermatology Life Quality Index (DLQI) Test
}

\author{
Alessia Villani - Gabriella Fabbrocini • Milena Cappello • \\ Claudia Costa $\cdot$ Massimiliano Scalvenzi
}

Received: April 8, 2019 / Published online: June 4, 2019

(C) The Author(s) 2019

\begin{abstract}
Introduction: Non-melanoma skin cancer (NMSC) is the most common type of human tumor, with an estimated five million new cases each year. NMSC has been described as having a major impact on the health-related quality of life of the patient. Vismodegib is a hedgehog pathway inhibitor therapy for patients who are affected by locally advanced basal cell carcinoma (laBCC) and metastatic basal cell carcinoma and are ineligible for surgery and/or radiotherapy. The objective of this study was to assess treatment-emergent adverse events reported by patients with advanced BCC who were undergoing hedgehog pathway inhibitor therapy with vismodegib, and to quantify their health-related quality of life using the Dermatology Life Quality Index (DLQI) questionnaire.
\end{abstract}

Enhanced Digital Features To view enhanced digital features for this article go to https://doi.org/10.6084/ m9.figshare.8153822.

Electronic supplementary material The online version of this article (https://doi.org/10.1007/s13555019-0303-4) contains supplementary material, which is available to authorized users.

A. Villani $(\bowtie) \cdot$ G. Fabbrocini · M. Cappello .

C. Costa $\cdot$ M. Scalvenzi

Dermatology Unit, Department of Clinical Medicine

and Surgery, University of Naples Federico II,

Naples, Italy

e-mail: ali.vil@hotmail.it
Methods: Patients with advanced and/or multiple basal cell carcinomas treated with vismodegib at the Non-Melanoma Skin Cancer Unit of the University of Naples Federico II (Italy) were consecutively enrolled. Each patient was evaluated every month until the end of the treatment cycle to assess adverse events related to the drug and the patient's quality of life.

Results: 48 patients (35 males and 13 females) with advanced BCC were included in the study. Muscle spasms, alopecia, and dysgeusia were the most frequently reported adverse events. 41 patients completed the DLQI questionnaire at the baseline visit and after 6 months of treatment. The average reported DLQI score decreased from a mean value of 5.7 at the baseline visit to 0.4 after 6 months of treatment. Conclusion: This is the first study to demonstrate a significant change in patient health-related quality of life from baseline to 6 months after hedgehog pathway inhibitor therapy initiation using the DLQI test. Interestingly, patients with BCC in visible areas such as the face or neck presented an overall DLQI score that was higher than that of patients with BCC located on the trunk and legs at the baseline visit, but the DLQI scores of these two groups were almost the same after 6 months of vismodegib therapy.

Keywords: Adverse events; Basal cell carcinoma; Quality of life; Sonic hedgehog inhibitor; Vismodegib 


\section{INTRODUCTION}

Non-melanoma skin cancer (NMSC) is the most common type of human tumor, with an estimated five million new cases each year. Although these tumors are associated with a very low mortality risk, they have been reported to have a major impact on the health-related quality of life of the patient [1]. This impact on quality of life derives from the symptomatology of the tumor, the various interventions applied, and the adverse events associated with these treatments [2]. Basal cell carcinoma (BCC) is the most common NMSC. Although surgery is the first-line therapy for BCC, some cases can progress to an advanced or-rarely-a metastatic state, meaning that nonsurgical approaches such as cryotherapy, adjuvant radiotherapy, and targeted therapy are required [3]. Vismodegib is a hedgehog pathway inhibitor therapy (HPIT) for patients who are affected by locally advanced basal cell carcinoma (laBCC) and metastatic basal cell carcinoma and are ineligible for surgery and/or radiotherapy [4]. Approximately $80 \%$ of BCCs occur in the head and neck region and may cause disfiguring skin changes that impact on the patient's body image and health-related quality of life (HRQoL) [5]. The aims of this clinical study were twofold: to assess treatment-emergent adverse events (AEs) reported by patients with advanced BCC who were undergoing hedgehog pathway inhibitor therapy (HPIT) with vismodegib, and to quantify their HRQoL using the Dermatology Life Quality Index (DLQI) questionnaire [6].

\section{METHODS}

This analysis included patients with advanced and/or multiple basal cell carcinomas treated with vismodegib at the Non-Melanoma Skin Cancer Unit of the University of Naples Federico II (Italy). Patients were consecutively enrolled in this descriptive study from November 2016 to November 2018. The approved dosage of vismodegib was $150 \mathrm{mg}$ per day. Demographic and clinical variables of each patient were analyzed. Each patient was evaluated every month until the end of the treatment cycle in order to assess adverse events related to the drug and their therapeutic response according to their degree of remission: either complete remission (CR; tumor size reduced by $>80 \%$ ), partial remission (PR; tumor size reduced by $50-80 \%$ ), stable disease (SD; tumor size reduced by $10-50 \%)$, or no response (NR; tumor size reduced by $<10 \%)$. To assess the specific impact of vismodegib therapy, the validated, short, self-administered DLQI questionnaire was administered at the baseline visit and after 6 months of treatment. The DLQI consists of 10 questions that require a response ranging from 0 (not at all) through 1 (a little) and 2 (a lot) to 3 (very much), with overall DLQI scores ranging from a minimum of 0 to a maximum of 30 . The higher the score, the more impaired the HRQoL. Analysis of the DLQI results focuses on six subcategories: (1) symptoms and feelings; (2) daily activities; (3) leisure; (4) work and school; (5) personal relations; and (6) treatment. The questionnaire was completed immediately before starting vismodegib therapy and again 6 months after treatment. The DLQI questionnaire is reported in the Electronic supplementary material (ESM).

\section{Compliance with Ethics Guidelines}

All procedures performed in studies involving human participants were in accordance with the ethical standards of the University of Naples Federico II and with the 1964 Declaration of Helsinki and its later amendments or comparable ethical standards. Informed consent was obtained from all individual participants included in the study.

\section{RESULTS}

Forty-eight patients (35 males and 13 females) with advanced BCC were included in the study. The median age was 74.5 years (ranging from 43 to 95 years). All patients had histologically confirmed advanced basal cell carcinoma, except for 2 female patients, 1 of whom was affected by Gorlin-Goltz syndrome and the other with a metastasizing BCC. Thirty $(62.5 \%)$ of the BCCs were located on the head or neck, 9 
(18.7\%) were on the trunk, 7 (9\%) were on the upper limbs, and $2(4.2 \%)$ were localized on the legs. At baseline, 22 patients $(45.8 \%)$ had an ulcerative BCC. The other reported clinical variants were multiple superficial and nodular BCCs $(29.2 \%)$, nodular BCCs $(20.8 \%)$, and a morpheaform variant in 2 patients. The average duration of treatment was 6 months (ranging from 3 to 10 months). Five patients $(10.4 \%)$ discontinued treatment after 2 months for scarce compliance, 1 patient discontinued after 6 months, having reported no response, and another patient discontinued after 2 months for multiple adverse events. Muscle spasms, dysgeusia, and alopecia were the most frequently reported adverse events. In particular, 38/48 patients reported muscle spasms; 38/48 reported dysgeusia; and 28/48 patients reported alopecia. The most frequently reported adverse events categorized by grade of severity are presented in Table 1. Furthermore, the majority of the patients reported mild to moderately severe adverse events; interestingly, most of the patients reported grade 2 dysgeusia and grade 1 muscle spasms. Alopecia, when present, was most frequently reported as a grade 3 adverse event. Forty-one patients completed the DLQI questionnaire at their baseline visit and after 6 months. The average reported DLQI score decreased from a mean value of 5.7 at the baseline visit to 0.4 after 6 months of treatment. Despite all of the adverse events, we found that, in general, the overall DLQI score significantly decreased. Scores in the "symptoms and feelings" subcategory significantly decreased from baseline to 6 months after treatment initiation. Scores in the other subcategories were generally very low. Patients with laBCCs in the head and neck regions had significantly higher DLQI scores before starting vismodegib therapy than

Table 1 The most frequently reported adverse events categorized by the grade of severity

\begin{tabular}{llll}
\hline Adverse event & Grade 1 & Grade 2 & Grade 3 \\
\hline Muscle spasms & 23 & 13 & 2 \\
Dysgeusia & 4 & 33 & 1 \\
Alopecia & 15 & 9 & 4 \\
\hline
\end{tabular}

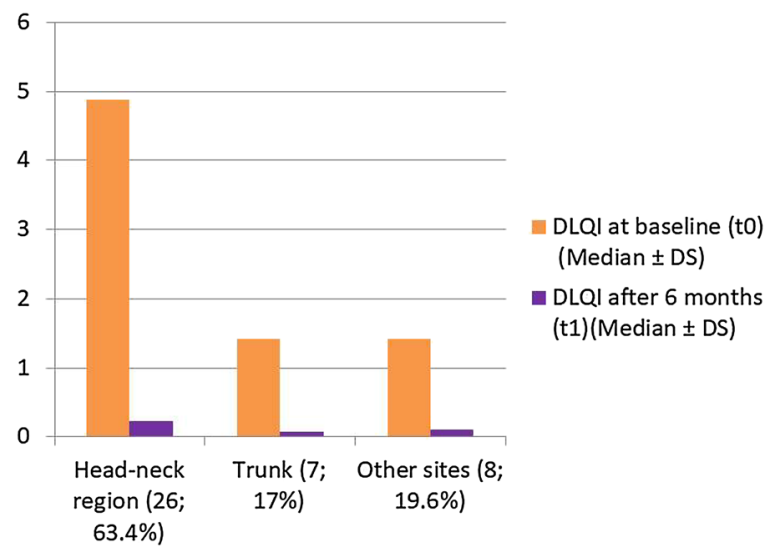

Fig. 1 DLQI values (median \pm DS) at baseline $(t 0)$ and after 6 months of treatment $(t 1)$ with vismodegib in patients with advanced BCCs categorized according to anatomical site $(n ; \%)$

patients who presented BCCs in other locations. DLQI values (median \pm DS) at baseline $(\mathrm{t} 0)$ and after 6 months of treatment (t1) with vismodegib in patients with advanced BCCs categorized according to anatomical site $(n ; \%)$ are presented in Fig. 1.

\section{DISCUSSION}

Basal cell carcinoma (BCC) is the most common form of skin malignancy worldwide, and accounts for about $80 \%$ of all nonmelanoma skin cancers $[1,2]$. Vismodegib, an oral sonic hedgehog signaling pathway inhibitor, has recently been approved by the Food and Drug Association for the treatment of locally advanced, metastatic, and recurrent basal cell carcinomas that are ineligible for surgery or radiotherapy [4]. Several studies have demonstrated the efficacy and safety of this small molecule which selectively connects to the Smoothened protein and blocks intracellular signaling, deactivating the hedgehog pathway and thus inhibiting tumor growth [7]. As reported in our results, muscle spasms, dysgeusia, weight loss, and alopecia are the most frequently described adverse events associated with vismodegib therapy. The timing and grade of severity of these events vary, but they 
affect patient quality of life and sometimes lead to treatment discontinuation [8]. The majority of our patients reported that mild to moderately severe AEs (grade 1 or 2) occurred in the first two months of therapy, except for alopecia, which occurred later and was almost always a severe event. In recent years, different dose reduction strategies during routine treatment have been proposed as a means to reduce $\mathrm{AE}$ severity and achieve the optimum duration of vismodegib treatment. Transitioning from a continuous to an intermittent regimen could be a way to avoid dropouts $[9,10]$. NMSC also has an appreciable impact on patient QOL, especially for the locally advanced and the metastasizing forms of BCC, due to the symptomatology involved and the potential for physical disfigurement, which usually compromises the psychological state of the patient [11]. In the present study, the mean overall DLQI score across 41 patients decreased from 5.7 at the baseline visit to 0.4 after 6 months of treatment, indicating that vismodegib had a positive effect on patient HRQOL according to Finlay and Khan [6]. The mean DLQI score (maximum possible score: 30) in a normal population usually ranges from 0 to 0.5 [12]. Two other studies have used the DLQI test to assess HRQOL in patients with BCCs who were treated with surgery, and those studies observed a significant decrease in DLQI score 3 months after surgery [13, 14]. Moreover, in 2012, a clinical study involving 25 patients with advanced BCCs who were treated with X-ray therapy showed a significant decrease in DLQI score to a normal level 3 months after treatment (from $1.2 \pm 1.5$ to $0.4 \pm 0.9$ ) [15]. In the literature, data on HRQOL in patients with advanced BCCs who were treated with vismodegib are limited. A phase 2 study of vismodegib safety, STEVIE (NCT01367665), reported the HRQoL outcomes in 730 patients with locally advanced BCCs and metastasizing BCCs. Skindex-16 and MD Anderson Symptom Inventory (MDASI) questionnaires were completed at baseline and at three subsequent visits, and the results pointed to an improvement in HRQoL in all vismodegib-treated patients [16]. Furthermore, in a recent study,
Gualdi et al. used a different test-the Pictorial Representation of Illness and Self Measure (PRISM) - to assess HRQoL in 10 patients treated with vismodegib, which was evaluated as the Self-Illness Separation (SIS) score. The authors reported that the average SIS score was inversely correlated to the average number of AEs, which tended to increase during the intermittent therapeutic regimen [17]. To the best of our knowledge, this is the first study to demonstrate a significant change in patient health-related quality of life from baseline to 6 months after hedgehog pathway inhibitor therapy initiation using the DLQI test. A significant decrease in the overall DLQI score was found, especially for the subcategory "symptoms and feelings," which significantly decreased from baseline to 6 months after treatment initiation, despite the occurrence of multiple AEs. Interestingly, although patients with BCCs in visible areas such as the face or neck presented a higher DLQI score at the baseline visit than patients with BCCs on the trunk and legs, the scores for the two groups were very similar after 6 months of vismodegib therapy. A number of authors argue that the DLQI may not be specific enough to accurately evaluate the quality of life in patients with basal cell carcinomas, as this kind of questionnaire is tailored to patients with chronic skin conditions such as eczema or psoriasis. This could indicate the need for a more disease-specific instrument that is validated for use in non-English-speaking countries [18].

\section{CONCLUSIONS}

In conclusion, although various adverse events have been linked to vismodegib treatment, it was found in the present work to be a safe and effective drug in almost all patients who presented with inoperable advanced BCCs, as it brought HRQOL levels back to those of healthy controls. Further studies are required to better manage hedgehog pathway inhibitor-related adverse events and to validate a specific assessment of the health-related quality of life in patients who are undergoing this treatment. 


\section{ACKNOWLEDGEMENTS}

Funding. No funding or sponsorship was received for this study or the publication of this article.

Authorship. All named authors meet the International Committee of Medical Journal Editors (ICMJE) criteria for authorship for this manuscript, take responsibility for the integrity of the work as a whole, and have given final approval to the version to be published.

Disclosures. Alessia Villani, Gabriella Fabbrocini, Milena Cappello, Claudia Costa, and Massimiliano Scalvenzi have nothing to disclose. Gabriella Fabbrocini is a member of the journal's Editorial Board.

Compliance with Ethics Guidelines. All procedures performed in studies involving human participants were in accordance with the ethical standards of the University of Naples Federico II and with the 1964 Declaration of Helsinki and its later amendments or comparable ethical standards. Informed consent was obtained from all individual participants included in the study.

Open Access. This article is distributed under the terms of the Creative Commons Attribution-NonCommercial 4.0 International License (http://creativecommons.org/licenses/ by-nc/4.0/), which permits any noncommercial use, distribution, and reproduction in any medium, provided you give appropriate credit to the original author(s) and the source, provide a link to the Creative Commons license, and indicate if changes were made.

\section{REFERENCES}

1. Gaulin C, Sebaratnam DF, Fernández-Peñas P. Quality of life in non-melanoma skin cancer. Australas J Dermatol. 2015;56(1):70-6.

2. Abedini R, Nasimi $M$, Noormohammad Pour $P$, Moghtadaie A, Tohidinik HR. Quality of life in patients with non-melanoma skin cancer: implications for healthcare education services and supports. J Cancer Educ. 2018. https://doi.org/10.1007/ s13187-018-1368-y.

3. Scalvenzi M, Villani A, Mazzella C, Cappello M, Salvatores GF, Costa C. Vismodegib treatment in a HIV positive patient on antiretroviraltherapy. Indian J Dermatol Venereol Leprol. 2018;84(6):758-60.

4. Basset-Séguin N, Hauschild A, Kunstfeld R, et al. Vismodegib in patients with advanced basal cell carcinoma: primary analysis of STEVIE, an international, open-label trial. Eur J Cancer. 2017;86:334-48.

5. Matthews BA, Rhee JS, Neuburg M, Burzynski ML, Nattinger AB. Development of the facial skin care index: a health-related outcomes index for skin cancer patients. Dermatol Surg. 2006;32(7):924-34.

6. Finlay AY, Khan GK. Dermatology Life Quality Index (DLQI) - a simple practical measure for routine clinical use. Clin Exp Dermatol. 1994;19(3): 210-6.

7. Girardi D, Barrichello A, Fernandes G, Pereira A. Targeting the Hedgehog pathway in cancer: current evidence and future perspectives. Cells. 2019;8(2): E153.

8. Fife $\mathrm{K}$, Herd $\mathrm{R}$, Lalondrelle $\mathrm{S}$, et al. Managing adverse events associated with vismodegib in the treatment of basal cell carcinoma. Future Oncol. 2017;13(2):175-84.

9. Woltsche N, Pichler N, Wolf I, Di Meo N, Zalaudek I. Managing adverse effects by dose reduction during routine treatment of locally advanced basal cell carcinoma with the Hedgehog inhibitor vismodegib: a single centre experience. J Eur Acad Dermatol Venereol. 2019;33(4):e144-5.

10. Scalvenzi M, Costa C, Cappello M, Villani A. Reply to Woltsche $\mathrm{N}$. et al. Managing adverse effects by dose reduction during routine treatment of locally advanced basal cell carcinoma with the Hedgehog inhibitor vismodegib: a single center experience. J Eur Acad Dermatol Venereol. 2019;33(4):e145-7.

11. Matthews BA, Rhee JS, Neuburg M, Burzynski ML, Nattinger AB. Development of the facial skin care index: a health-related outcomes index for skin cancer patients. Dermatol Surg. 2006;32(7):924-34 (discussion 934).

12. Lewis V, Finlay AY. 10 years experience of the Dermatology Life Quality Index (DLQI). J Investig Dermatol. 2004;9(2):169-80.

13. Blackford S, Roberts D, Salek MS, Finlay A. Basal cell carcinomas cause little handicap. Qual Life Res. 1996;5(2):191-4. 
14. Rhee JS, Matthews BA, Neuburg M, Smith TL, Burzynski M, Nattinger AB. Skin cancer and quality of life: assessment with the dermatology life quality index. Dermatol Surg. 2004;30(4 Pt 1):525-9.

15. Skiveren J, Mikkelsen MR, Daugbjerg H, Wulf HC. Skin reactions and quality of life after X-ray therapy of basal cell carcinoma. J Skin Cancer. 2012;2012:825095.

16. Hansson J, Bartley K, Karagiannis T, et al. Assessment of quality of life using Skindex-16 in patients with advanced basal cell carcinoma treated with vismodegib in the STEVIE study. Eur J Dermatol. 2018;28(6):775-83.
17. Gualdi G, Moro R, Regina V, Caravello S, Monari P, Calzavara-Pinton PG. PRISModegib: the use of the PRISM test to assess the health-related quality of life of patients with locally advanced basal cell carcinoma undergoing Hedgehog pathway inhibitor therapy. Br J Dermatol. 2019. https://doi.org/10. 1111/bjd.17754.

18. Shah M, Coates M. An assessment of the quality of life in older patients with skin disease. Br J Dermatol. 2006;154(1):150-3. 